\title{
EMBEDDING THE FREE GROUP $F(X)$ INTO $F(\beta X)$
}

\author{
TEMPLE H. FAY, M. RAJAGOPALAN AND \\ BARBARA V. SMITH-THOMAS
}

\begin{abstract}
We show that for a Tychonoff space, $X$ and the canonical embedding $\beta_{X}: X \rightarrow \beta X$, the induced homomorphism $F \beta_{X}: F(X) \rightarrow F(\beta X)$ is an embedding between the free topological groups when $X$ has the property that $X^{n}$ is pseudocompact for all $n>1$. An application of this result is if $X$ is such a space and $\beta X$ is 0-dimensional, then $F(X)$ is 0-dimensional.
\end{abstract}

Introduction. Let $X$ be a Tychonoff space and $\beta_{X}: X \rightarrow \beta X$ the canonical dense embedding of $X$ into its Stone-Čech compactification. In 1976, Hardy, Morris, and Thompson [7] raised the question of when the induced homomorphism $F \beta_{X}$ : $F(X) \rightarrow F(\beta X)$ is an embedding. Here $F(-)$ denotes the free topological group functor. $F \beta_{X}$ is always continuous and injective, but need not be an embedding; a striking example of this is given by choosing $X=\mathbf{R}$.

In this paper we show that $F \beta_{X}$ is an embedding when $X$ has the property that $X^{n}$ is pseudocompact for all $n \geqslant 1$. More generally, if $X$ is $C^{*}$-embedded in $Y$ and $X^{n}$ is pseudocompact for all $n \geqslant 1$, then $F(X)$ is embedded as a topological group in $F(Y)$. These theorems extend Ordman's result that if $X$ is a compact subspace of $Y$, then $F(X)$ is embedded in $F(Y)$ [11]. Recently E. Nummela has improved our result. Using free uniform groups he has shown that it suffices to assume only that $X$ itself, rather than all finite powers of $X$, is pseudocompact [10].

The class of spaces $X$ with the property that $X^{n}$ is pseudocompact is fairly extensive. Examples include the Tychonoff plank, $\beta \mathbf{N} \backslash\{p\}$ where $p$ is a $P$-point of $\beta \mathbf{N}$, the ordinal space $[1, \Omega)$, and of course any compact space.

As an application of this result, we show that if $X$ has the property that $X^{n}$ is pseudocompact for all $n \geqslant 1$, and is strongly 0 -dimensional, then $F(X)$ is 0 -dimensional.

Preliminaries. The free topological group $F(X)$ over a pointed space $(X, e)$ was introduced by Graev [6]; algebraically it is the free group over $X \backslash\{e\}$, and it carries the finest group topology making the inclusion of $X$ into $F(X)$ continuous (this inclusion carries $e$ to the group identity). It turns out that $F(X)$ is independent of the choice of the base point and the inclusion of $X$ into $F(X)$ is a closed embedding exactly when $X$ is a Tychonoff space. In this case $F(X)$ is also Tychonoff and, consequently, in this paper we assume all spaces to be Tychonoff.

Received by the editors July 30, 1980 and, in revised form, April 24, 1981; presented to the Society, November 15, 1980.

1980 Mathematics Subject Classification. Primary 22A05, 54D30, 54D35.

Key words and phrases. Free topological group, pseudocompact, Stone-Čech compactification, Tychonoff space. 
The nature of the topology on $F(X)$ has proved to be rather intractible; however, good results have been obtained in the case that $X$ is a $k_{\omega}$-space. Recall that a space $X$ is a $k_{\omega}$-space provided it is a weak union of countably many compact subsets; see $[3,9,11]$. In this case $F(X)$ is also $k_{\omega}$ and the topology on $F(X)$ is more clearly understood; in particular $F(X)$ is the weak union of the subsets $F(X)_{n}$ defined later in this paper [11].

The free topological group over $X$ is free in the classical sense. That is given any continuous $f: X \rightarrow G$, where $G$ is a topological group and $f(e)=e_{G}$, there is a unique continuous group homomorphism $\hat{f}$ making the following diagram commute:

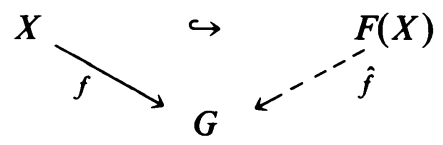

Thus $F$ is functorial; that is, if $f: X \rightarrow Y$ is a base point preserving continuous function then $f$ lifts to a continuous group homomorphism $F(f): F(X) \rightarrow F(Y)$. In particular if $i$ is the inclusion mapping of $X$ into $Y$ then $i$ lifts to a continuous injective group homomorphism $F(i): F(X) \rightarrow F(Y)$. (We always choose the base point of $Y$ to be the base point of $X$, since $F(X)$ and $F(Y)$ are independent of choice of base point.)

There is another notion of free topological group due to Markov [8]. The Markov free topological group $F_{M}(X)$ over $X$ has for underlying group the free group on $X$ itself (rather than $X \backslash\{e\}$ ). The description of the topology of $F_{M}(X)$ is similar to that of $F(X)$ [13]. In fact, $F_{M}(X)$ is isomorphic to $F(X \cup\{e\})$ where $e$ is not an element of $X$; thus without loss of generality we restrict ourselves to considering the Graev free group.

Lifting the embedding. It has been known for a long time that $F(i): F(X) \rightarrow$ $F(Y)$ need not be an embedding. For a fairly simple example take $X=\mathbf{N}$ and $Y=\alpha \mathbf{N}$, the one point compactification of $\mathbf{N} ; F(\mathbf{N})$ is discrete but its image in $F(\alpha \mathbf{N})$ is not [13]. In [7] Hardy, Morris, and Thompson showed that if $X$ is a noncompact $k_{\omega}$-space then the topology $F\left(\beta_{X}\right)(F(X))$ inherits from $F(\beta X)$ is not the free topology. In particular $F\left(\beta_{\mathbf{R}}\right): F(\mathbf{R}) \rightarrow F(\beta \mathbf{R})$ is not an embedding.

Until now the best results in the positive direction have been that if $X=C$ is a compact subspace of $Y$ then $F(i): F(C) \rightarrow F(Y)$ is an embedding [11], and that if $X$ is a closed subspace of a normal space $Y$ then $F(i): F(X) \rightarrow F(Y)$ is an embedding [10]. In this paper we establish

MAIN THEOREM. If $X$ is a Tychonoff space with the property that $X^{n}$ is pseudocompact for all $n \geqslant 1$ then the subgroup of $F(\beta X)$ generated by $X$ is isomorphic, as a topological group, to $F(X)$.

We need the following preliminary definitions, lemmas and theorems.

Notation. Every element $w$ of $F(X)$ has a reduced representation $x_{1}^{\varepsilon_{1}} x_{2}^{\varepsilon_{2}} \cdots x_{n}^{\varepsilon_{n}}$ where, for each $i, \varepsilon_{i}= \pm 1$ and $x_{i} \in X$. We call $n$ the length of $w$, unless $w=e$. If $w=e$ we say $w$ has length 0 . Thus if the length of $w$ is $>1, e$ does not appear in 
its reduced representation. We denote the (closed [6]) subset of $F(X)$ consisting of all words of length $\leqslant n$ by $F(X)_{n}$.

Definition 1. Let $n \in \mathbf{N}$; let $X_{n}^{*}=X^{n} \times\{-1,1\}^{n}$. Thus an element of $X_{n}^{*}$ is a pair $\left(\left(x_{1}, x_{2}, \ldots, x_{n}\right),\left(\varepsilon_{1}, \varepsilon_{2}, \ldots, \varepsilon_{n}\right)\right)$ where each $x_{i} \in X$ and each $\varepsilon_{i}= \pm 1$. We say that two elements $\left(\left(x_{1}, \ldots, x_{n}\right),\left(\varepsilon_{1}, \ldots, \varepsilon_{n}\right)\right)$ and $\left(\left(y_{1}, \ldots, y_{n}\right),\left(\delta_{1}, \ldots, \delta_{n}\right)\right)$ are related if $x_{1}^{\varepsilon_{1}} \cdots x_{n}^{\varepsilon_{n}}$ and $y_{1}^{\delta_{1}} \cdots y_{n}^{\delta_{n}}$ represent the same element of $F(X)$. Call this equivalence relation $R_{n}$. Similarly let $(\beta X)_{n}^{*}=(\beta X)^{n} \times\{-1,1\}^{n}$ and denote by $\tilde{R}_{n}$ the relation on $(\beta X)_{n}^{*}$ whose definition is obtained by replacing $F(X)$ by $F(\beta X)$. Note that $R_{n}=\tilde{R}_{n} \cap X_{n}^{*} \times X_{n}^{*}$.

Definition 2. Let $X_{n}^{*}$ and $(\beta X)_{n}^{*}$ be as in Definition 1. Let $f: X_{n}^{*} \rightarrow \mathbf{R}$ be a real-valued function; $f$ is said to respect $R_{n}$ if it is constant on equivalence classes $\left(\bmod R_{n}\right)$. Similarly $g:(\beta X)_{n}^{*} \rightarrow \mathbf{R}$ respects $\tilde{R}_{n}$ if it is constant on equivalence classes $\left(\bmod \tilde{R}_{n}\right)$.

Definition 3. For each $n \in \mathrm{N}$, define $m_{n}: X_{n}^{*} \rightarrow F(X)$ by

$$
m_{n}\left(\left(x_{1}, \ldots, x_{n}\right),\left(\varepsilon_{1}, \ldots, \varepsilon_{n}\right)\right)=x_{1}^{e_{1}} \ldots x_{n}^{e_{n}} \text {, }
$$

and define $\tilde{m}_{n}:(\beta X)_{n}^{*} \rightarrow F(\beta X)$ by

$$
\tilde{m}_{n}\left(\left(z_{1}, \ldots, z_{n}\right),\left(\varepsilon_{1}, \ldots, \varepsilon_{n}\right)\right)=z_{1}^{\varepsilon_{1}} \ldots z_{n}^{\varepsilon_{n}} .
$$

Note that the image of $m_{n}$ is $F(X)_{n}$, that the image of $\tilde{m}_{n}$ is $F(\beta X)_{n}$, that two elements $a$ and $b$ of $X_{n}^{*}$ are related by $R_{n}$ if and only if $m_{n}(a)=m_{n}(b)$, and that two elements $\tilde{a}$ and $\tilde{b}$ of $(\beta X)_{n}^{*}$ are related by $\tilde{R}_{n}$ if and only if $\tilde{m}_{n}(\tilde{a})=\tilde{m}_{n}(\tilde{b})$.

Observe that $m_{n}$ and $\tilde{m}_{n}$ are both continuous; we only argue that $m_{n}$ is continuous. The proof for $\tilde{m}_{n}$ is similar. First note that $m_{1}: X \times\{-1,1\} \rightarrow F(X)$ is continuous since $X$ and $\left\{x^{-1} \mid x \in X\right\}$ are both subspaces of $F(X)$ and the only action of $m_{1}$ is to identify $e^{1}$ with $e^{-1}$. Then $m_{n}$ is essentially the composition of the product of $m_{1}$ with itself $n$ times followed by multiplication in $F(X)$.

LEMMA 4. If $X^{n}$ is pseudocompact then $F(\beta X)_{n}$ is the Stone-Čech compactification of $F(X)_{n}$ via the canonical $j_{n}: F(X)_{n} \rightarrow F(\beta X)_{n}$.

Proof. Consider the following commutative diagram:

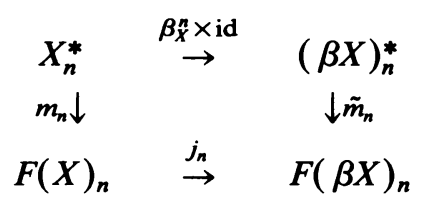

where $\beta_{X}: X \rightarrow \beta X$ is the canonical inclusion and $j_{n}$ is the restriction to $F(X)_{n}$ of $F\left(\beta_{X}\right)$. Note that $\beta_{X}^{n} \times$ id is an embedding and $j_{n}$ is continuous and one-to-one, and has dense range. Further $\tilde{m}_{n}$ is a quotient map since it has compact domain, so if $g^{\prime}:(\beta x)_{n}^{*} \rightarrow \mathbf{R}$ is continuous and respects $\tilde{R}_{n}$ then there is a continuous $g$ : $F(\beta X)_{n} \rightarrow \mathbf{R}$ such that $g^{\prime}=g \circ \tilde{m}_{n}$.

Now note that if $f^{\prime}: X_{n}^{*} \rightarrow \mathbf{R}$ is continuous and respects $R_{n}$ and if $g^{\prime}:(\beta X)_{n}^{*} \rightarrow \mathbf{R}$ is a continuous extension of $f^{\prime}$ then $g^{\prime}$ respects $\tilde{R}_{n}$. For suppose $a=\left(\left(x_{1}, \ldots, x_{n}\right)\right.$, $\left.\left(\varepsilon_{1}, \ldots, \varepsilon_{n}\right)\right)$ and $b=\left(\left(y_{1}, \ldots, y_{n}\right),\left(\delta_{1}, \ldots, \delta_{n}\right)\right)$ with $a$ and $b$ in $\tilde{R}_{n} \backslash R_{n}$. If $\tilde{m}_{n}(a)=\tilde{m}_{n}(b)$ has no occurrences of elements of $\beta X \backslash X$ in its reduced representation then there exist two nets $\left\{a_{\alpha}\right\},\left\{b_{\alpha}\right\}$ in $X_{n}^{*}$, on the same directed set, with 
$a_{\alpha} \rightarrow a$ and $b_{\alpha} \rightarrow b$ and with every term in either net having reduced representation $\tilde{m}_{n}(a)=\tilde{m}_{n}(b)$, since the $x_{i}$ 's in $a$, and the $y_{i}$ 's in $b$ which are from $\beta X \backslash X$ must occur in cancelling pairs. It follows that $g^{\prime}(a)=g^{\prime}(b)$. If $\tilde{m}_{n}(a)=\tilde{m}_{n}(b)$ has some elements of $\beta X \backslash X$ in its reduced representation, then we can find for each such element a net in $X$ (all on the same directed set if more than one) converging to that element. Replacing the noncancelling elements of $\beta X \backslash X$ in both $a$ and $b$ by the corresponding nets from $X$ chosen above, we obtain nets $\left\{a_{\alpha}\right\} \rightarrow a$ and $\left\{b_{\alpha}\right\} \rightarrow b$ with the property that, for all $\alpha, \tilde{m}_{n}\left(a_{\alpha}\right)=\tilde{m}_{n}\left(b_{\alpha}\right)$ and whose reduced representations contain no letters from $\beta X \backslash X$. Since we have already shown that, for all $\alpha, g^{\prime}\left(a_{\alpha}\right)=g^{\prime}\left(b_{\alpha}\right)$ we can conclude that $g^{\prime}(a)=g^{\prime}(b)$.

Finally let $f: F(X)_{n} \rightarrow \mathbf{R}$ be continuous; $f$ is necessarily bounded since $X_{n}^{*}$ is pseudocompact and $m_{n}$ is onto. From Glicksburg's Theorem [5] we know that $(\beta X)_{n}^{*}=\beta\left(X_{n}^{*}\right)$ so $f^{\prime}=f \circ m_{n}: X_{n}^{*} \rightarrow \mathbf{R}$ extends continuously to some $g^{\prime}:(\beta X)_{n}^{*} \rightarrow$ R. By the above argument $g^{\prime}$ respects $\tilde{R}_{n}$; hence there exists a continuous $g$ : $F(\beta X)_{n} \rightarrow \mathbf{R}$ such that $g \circ \tilde{m}_{n}=g^{\prime}$. Since $m_{n}$ is onto, it follows that $f=g \circ j_{n}$, that is $g$ extends $f$ continuously. Thus $\beta\left(F(X)_{n}\right)=F(\beta X)_{n}$.

LEMMA 5. If each $X^{n}$ is pseudocompact then every bounded continuous function $f$ : $F(X) \rightarrow \mathbf{R}$ extends to a bounded continuous function $g: F(\beta X) \rightarrow \mathbf{R}$.

Proof. Without loss of generality we may assume $f: F(X) \rightarrow[0,1]$. For each $n$ let $f_{n}: F(X)_{n} \rightarrow[0,1]$ be the restriction of $f$ to $F(X)_{n}$. By the preceding lemma $f_{n}$ extends to a continuous function $g_{n}: F(\beta X)_{n} \rightarrow[0,1]$. Moreover, since each $f_{n+1}$ extends $f_{n}$ it follows that each $g_{n+1}$ extends $g_{n}$. Now, since $\beta X$ is compact and $F(\beta X)$ is a $k_{\omega}$-space with $k_{\omega}$-decomposition $\cup_{n=1}^{\infty} F(\beta X)_{n}$ [1], the function $g$ : $F(\beta X) \rightarrow[0,1]$ which coincides with each $g_{n}$ on $F(\beta X)_{n}$ is continuous. This $g$ clearly extends $f$.

MAIN THEOREM. If each $X^{n}$ is pseudocompact then $F\left(\beta_{X}\right): F(X) \rightarrow F(\beta X)$ is an embedding.

Proof. From the preceding lemma we see that each bounded continuous $f$ : $F(X) \rightarrow \mathbf{R}$ extends to a continuous bounded $g: F(\beta X) \rightarrow \mathbf{R}$ and thus extends to a continuous $\hat{g}: \beta(F(\beta X)) \rightarrow \mathbf{R}$. Clearly the composition $F(X) \rightarrow^{F\left(\beta_{x}\right)} F(\beta X) \hookrightarrow$ $\beta(F(\beta X))$ has dense range so $\beta(F(\beta X))=\beta(F(X))$. It follows that $F\left(\beta_{X}\right)$ is an embedding, since all the spaces are Tychonoff.

COROLlaRY 6. If $X$ is $C^{*}$-embedded in $Y$ and, for each $n>1, X^{n}$ is pseudocompact, then $F(X)$ is embedded as a topological subgroup of $F(Y)$.

Proof. Suppose $X$ is $C^{*}$-embedded in $Y$; then $\mathrm{cl}_{\beta Y}(X) \cong \beta X$ [4]. Consider the following commutative diagram:

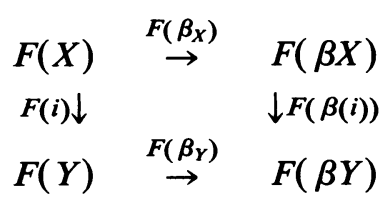


Each of the maps is $F$ of an inclusion. From Ordman's result stated above $F(\beta(i))$ is an embedding; we have just shown that $F\left(\beta_{X}\right)$ is an embedding. It follows that $F(i)$ is an embedding.

Some examples. Our first example shows that in Corollary 6 some hypothesis is needed concerning the way $X$ is embedded in $Y$. Let $Y$ be the union of two copies of $\Omega+1$, say $[0, \Omega] \cup[0, \Omega]^{*}$ with $\Omega$ and $\Omega^{*}$ identified. Let $X=Y \backslash\{\Omega\}$ and let both have $e=0$. Then $X$ is the disjoint union of two copies of $\Omega$, so $X^{n}$ is countably compact for all $n$. However, $X$ is not $C^{*}$-embedded in $Y$, for the function $f: X \rightarrow \mathbf{R}$ which is 0 on the first copy of $\Omega$ in $X$ and is 1 on the second copy of $\Omega$ does not extend to $Y$. This same function can be used to show that $F(X)$ is not a subspace of $F(Y)$ via the lifted embedding. For, the net $w_{\alpha}=\alpha^{*} \cdot \alpha^{-1}$ clearly converges to $e$ in $Y$, and thus also converges to $e$ in the subspace topology the subgroup generated by $X$ inherits from $F(Y)$. On the other hand, the function $f$ lif ts to a unique group homomorphism $\hat{f}: F(X) \rightarrow \mathbf{R}$. For every $\alpha, f\left(w_{\alpha}\right)=1 \neq 0$ so $w_{\alpha}$ cannot converge to $e$ in $F(X)$.

Some examples to which our Main Theorem does apply are: Let $X$ be the ordinal space $\Omega$; then $\beta X=\Omega+1$ and $F(\Omega)$ is a subspace of $F(\Omega+1)$. Let $X$ be the Tychonoff plank $T$; then $\beta X=(\Omega+1) \times(\omega+1)$ and $F(X)$ is a subspace of $F(\beta X)$. Let $X=\beta \mathbf{N} \backslash\{p\}$ where $p$ is a $P$-point of $\beta \mathbf{N}$; then $\beta X=\beta \mathbf{N}$ and $F(X)$ is a subspace of $F(\beta \mathbf{N})$.

An application. In [2] we show that if $X$ is 0 -dimensional then $F(X)$ is totally disconnected. It follows that if $X$ is a $k_{\omega}$-space, $X$ is 0 -dimensional if and only if $F(X)$ is 0 -dimensional. However, it is not known in general if $X$ is 0-dimensional whether $F(X)$ need be 0-dimensional. For example, it is unknown if $F(\mathbf{R} \backslash \mathbf{Q})$ is 0 -dimensional. As an application of our Main Theorem, we enlarge the class of spaces $X$ for which it is known that $F(X)$ is 0-dimensional.

THEOREM. If $X$ is a strongly 0-dimensional space with the property that $X^{n}$ is pseudocompact for all $n \geqslant 1$, then $F(X)$ is 0-dimensional.

Proof. Since $X$ is strongly 0 -dimensional, $\beta X$ is 0 -dimensional and thus $F(\beta X)$ is 0-dimensional. $F(X)$ being embedded in $F(\beta X)$ is necessarily 0-dimensional.

Thus the free group $F(X)$ is 0 -dimensional if $X$ is the Tychonoff plank, $\beta \mathbf{N} \backslash\{p\}$ where $p$ is a $P$-point of $\beta N$, or the ordinal space $[1, \Omega)$.

\section{REFERENCES}

0. C. R. Borges, Free topological groups, J. Austral. Math. Soc. Ser. A 23 (1977), 360-365.

1. T. H. Fay, E. T. Ordman and B. V. Smith-Thomas, The free topological group over the rationals, Gen. Topology Appl. 10 (1979), 33-47.

2. T. H. Fay, M. Rajagopalan and B. V. Smith-Thomas, Free groups, free products, and 0-dimensionality, Houston J. Math. (to appear).

3. S. P. Franklin and B. V. Smith-Thomas, $A$ survey of $\boldsymbol{k}_{\omega}$-spaces, Topology Proc. 2 (1977), 111-124.

4. L. Gillman and M. Jerrison, Rings of continuous functions, Van Nostrand, Princeton, N. J., 1960.

5. I. Glicksburg, Stone-Cech compactifications of products, Trans. Amer. Math. Soc. 90 (1959), 369-382.

6. M. I. Graev, Free topological groups, Izv. Akad. Nauk SSSR Ser. Mat. 12 (1948), 279-324; English transl. in Amer. Math. Soc. Transl., no. 35 (1951); reprinted in Amer. Math. Soc. Transl. (1) 8 (1962), 305-364. 
7. J. P. L. Hardy, S. A. Morris and H. B. Thompson, Applications of the Stone-Cech compactification to free topological groups, Proc. Amer. Math. Soc. 55 (1976), 160-164.

8. A. A. Markov, On free topological groups, C. R. (Doklady) Akad. Sci. URSS (N. S.) 31 (1941), 299-301; Izv. Akad. Nauk SSSR Ser. Mat. 9 (1945), 3-64; English transl. in Amer. Math. Soc. Transl., no. 30 (1950), 11-88; reprinted in Amer. Math. Soc. Transl. (1) 8 (1962), 195-275.

9. S. Morris, E. T. Ordman and H. B. Thompson, The topology of free products of topological groups, Proc. Second Internat. Conf. Group Theory (Canberra, 1973), Lecture Notes in Math., vol. 372, Springer-Verlag, Berlin and New York, 1974, pp. 504-515.

10. E. Nummela, Uniform free topological groups and Samuel compactifications, Topology Appl. (to appear).

11. E. T. Ordman, Free k-groups and free topological groups, Gen. Topology Appl. 5 (1975), 205-219.

12. P. Samuel, On universal mappings and free topological groups, Bull. Amer. Math. Soc. 54 (1948), 591-598.

13. B. V. S. Thomas, Free topological groups, Gen. Topology Appl. 4 (1974), 51-72.

Department of Mathematics, University of Southern Mississippi, Hattiesburg, MississipPI 39401 (Current address of T. H. Fay)

Department of Mathematics, California State University, Chico, California 95929

Department of Mathematics, University of Alabama in Birmingham, Birmingham, Alabama 35294 43606

Current address (M. Rajagopalan): Department of Mathematics, University of Toledo, Toledo, Ohio

Current address (B. V. Smith-Thomas): School of Information \& Computer Science, Georgia Institute of Technology, Atlanta, Georgia 30332 